\title{
An unusual case of appendicitis
}

\section{Cora Constantinescu MD, Joseph Vayalumkal MD, Dina Fisher MD MSc}

$\mathrm{A}$ 15-year-old boy, who had immigrated to Canada from the Philippines at the age of 11 , presented to the emergency department with fever and acute onset of periumbilical abdominal pain that migrated to the right lower quadrant over 24 hours. Before this, he had experienced four months of intermittent abdominal pain, which occurred about weekly, but no fever or weight loss. On examination, he was focally tender over the McBurney point. $\mathrm{He}$ had a normal hemoglobin level of 150 (normal 137-180) g/L, and elevated white blood count of 12.8 (normal 4.0-11.0) $\times 10^{9} / \mathrm{L}$ and neutrophil count of 10 (normal 2.0-8.0) $\times 10^{9} / \mathrm{L}$. Ultrasonography of the abdomen showed an enlarged appendix leading into a bi-lobed, complex right lower quadrant collection. He underwent a laparoscopic appendectomy for acute perforated appendicitis. At the time of surgery, the appendix was found to be thickened and inflamed. A retroiliac abscess was also found, filled with thick creamy contents that resembled cottage cheese. The appendix was removed and the abscess drained. The patient was given ampicillin, gentamicin and metronidazole for six days and then amoxicillin-clavulanate for another five days. His recovery was uneventful. When seen in follow-up six weeks postoperatively, the patient was doing well and had no specific symptoms. The pathology report of the appendix, however, described florid necrotizing granulomatous inflammation with extension into the mucosa, and tuberculosis (TB) was suspected (Figure 1).

The patient was referred to the infectious diseases clinic for further assessment. He described a mild cough 48 hours before the surgery, which recurred five to six weeks postoperatively. His review of systems was otherwise unremarkable, and he had no history of chronic fever or night sweats. Before emigrating from the Philippines four years earlier, he had lived with his grandmother, who had received treatment for pulmonary TB seven years earlier.

Given our strong suspicion of TB, we performed a Mantoux test, which was strongly positive at $17 \mathrm{~mm}$. Chest radiography showed ill- defined parenchymal opacities and multiple bilateral upper-lobe nodules scattered throughout both lung fields (Figure 2). An induced sputum sample tested positive for acid fast bacilli.

We diagnosed multifocal tuberculosis. The patient was referred to the regional TB clinic and started on four-drug therapy with isoniazid, rifampin, ethambutol and pyrazinamide. He was confirmed to be HIV-negative. Mycobacterium tuberculosis was isolated from his sputum cultures. Susceptibility tests showed that the isolate was resistant to isoniazid, and his treatment was tailored appropriately. Results of repeat sputum smear and culture were negative within two months, and repeat chest radiography was normal within five months. He tolerated his treatment well and successfully completed nine months of therapy with complete resolution of symptoms.

\section{Discussion}

The most recent TB report from the Public Health Agency of Canada showed a TB incidence of 4.6 per 100000 population in Canada. ${ }^{1}$ Of the reported TB cases, $66 \%$ occurred in foreign-born individuals, 21\% in Canadian-born Aboriginal people and $12 \%$ in Canadian-born non-Aboriginal people. ${ }^{1}$ The World Health Organization has identified 22 developing countries with a high burden of TB that account for about $80 \%$ of all new TB cases arising each year. ${ }^{2}$ In the case described, the patient emigrated from a TB-endemic region and had a known family contact who had received treatment for TB in the past. Immigrants to Can-

\section{KEY POINTS}

- Appendicular tuberculosis (TB) is rare, but demographic, intraoperative and postoperative features can point to the diagnosis.

- Clinical features may include abdominal pain, fever and non-liver related ascites; pathologic features include granulomas and caseous necrosis.

- The treatment for appendicular TB is surgical (i.e., appendectomy) as well as medical (i.e., with antituberculous drugs).

- All patients with appendicular or any other extrapulmonary TB need to be assessed for pulmonary TB.
Competing interests: None declared.

This article has been peer reviewed.

The authors have obtained patient consent.

Correspondence to: joseph.vayalumkal @ albertathealthservices.ca

CMAJ 2014. DOI:10.1503 /cmaj.131126 Joseph Vayalumkal, 


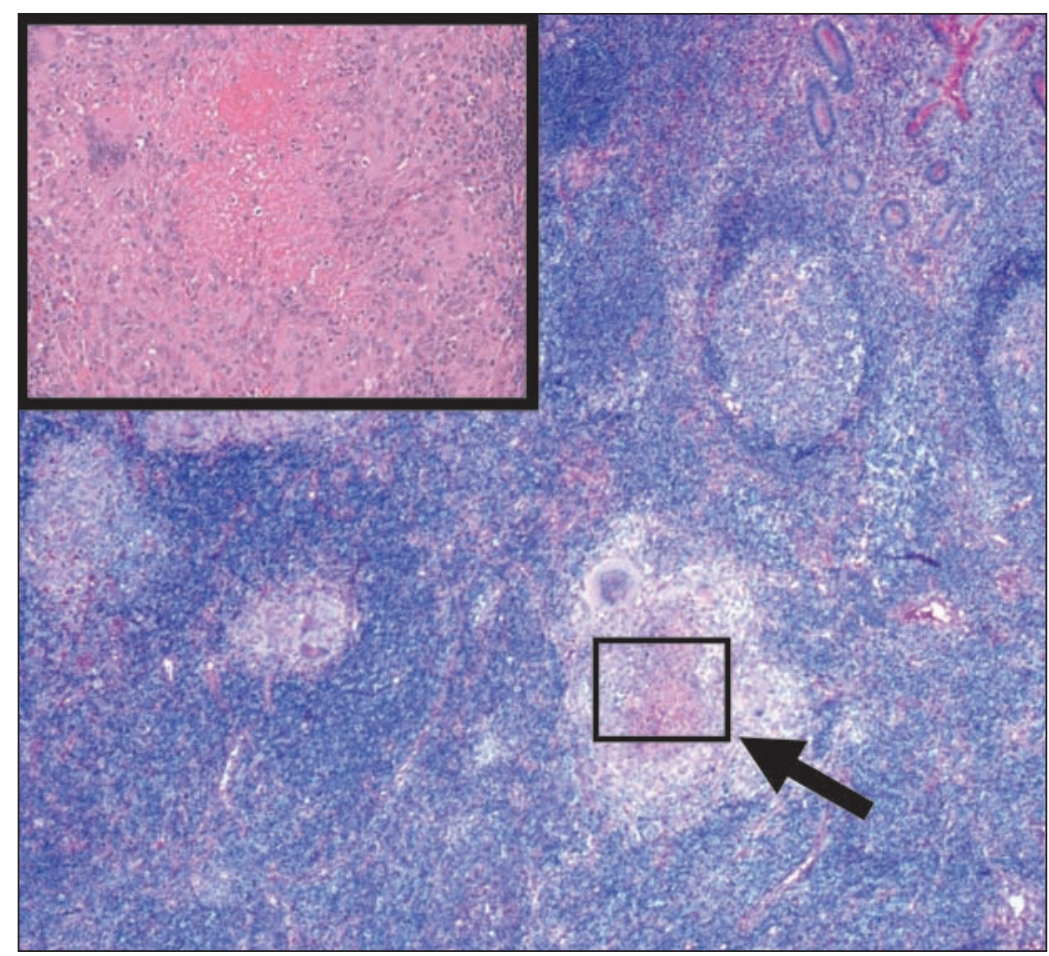

Figure 1: Pathology specimen of appendiceal mucosa from a 15-year-old boy with suspected tuberculous appendicitis showing granulomatous inflammation (hematoxylin-eosin, original magnification $\times 4$ ). Insert: Tuberculous granuloma showing an area of central caseous necrosis surrounded by multiple Langhanstype giant cells, epithelioid histiocytes and lymphocytes (hematoxylin-eosin, original magnification $\times 40$ ).

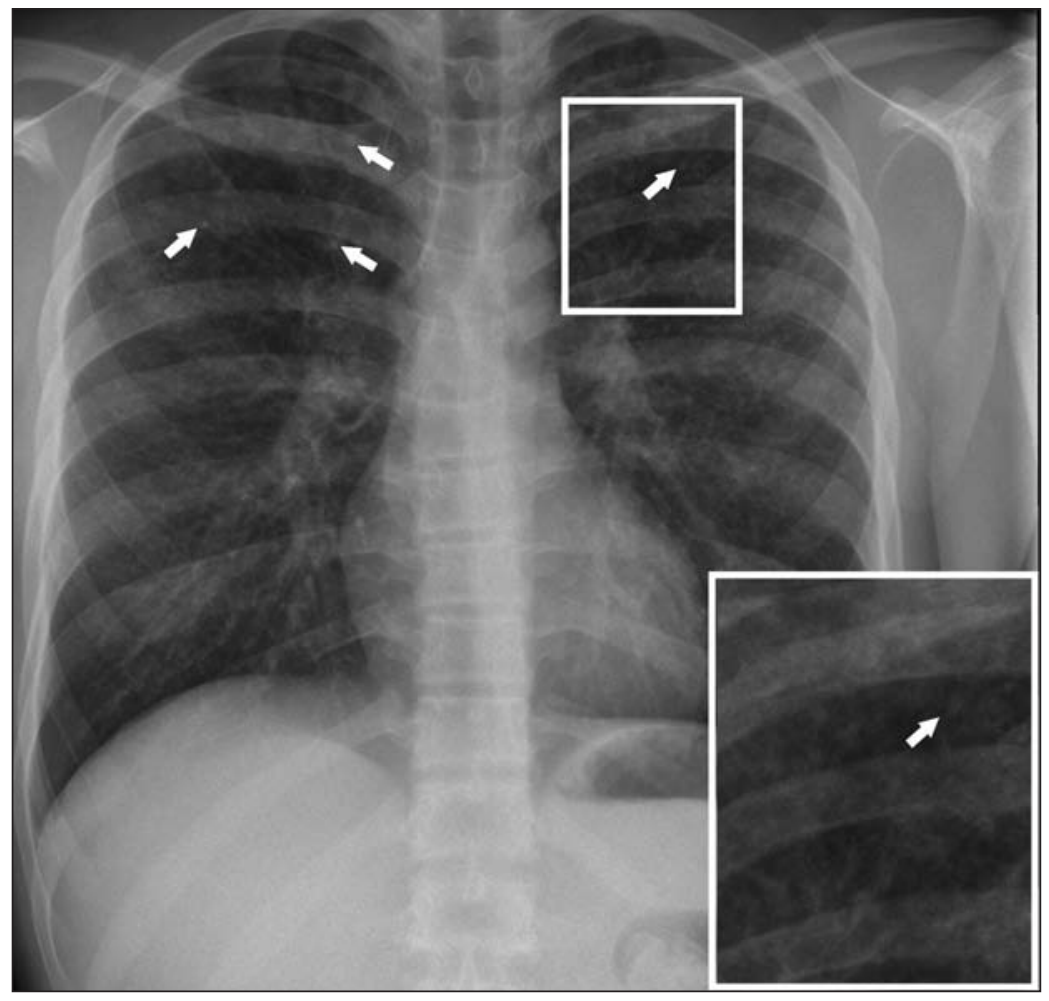

Figure 2: Chest radiograph showing ill-defined parenchymal opacities and multiple bilateral upper-lobe nodules (the latter highlighted by the arrows). Insert: magnification of one of the left upper-lobe nodules. ada are screened pre-arrival for active TB infection with chest radiography, followed by sputum culture if radiographic results are abnormal. ${ }^{3}$ However, children under 11 years of age do not undergo pre-arrival radiographic screening. ${ }^{3}$ This is of particular concern, because TB in children is often more severe or rapidly progressive, and is also more often extrapulmonary and therefore may be more difficult to diagnose. ${ }^{4}$ Our patient was under 11 years of age when applying for immigration and had his 11th birthday just before arrival in Canada. His mother confirmed that he did not have a pre-immigration chest radiograph, or any testing or treatment for TB in the past.

The species $M$. tuberculosis of the M. tuberculosis complex causes most cases of human TB. ${ }^{2}$ In immunocompetent older children and adults, the lifetime risk of progression from infection to TB disease is in the range of $5 \%-10 \%$. Among children, most progression to disease occurs in the first 2-12 months after the initial infection, and $60 \%-80 \%$ of cases are pulmonary $\mathrm{TB} .{ }^{5}$ In a retrospective case series involving children seen in two large hospital emergency departments, up to $25 \%$ of patients with TB presented with extrapulmonary TB, whereas disseminated disease accounted for only $1 \%-2 \%$ of cases, occurring mostly in children younger than five years of age and in those who were immunocompromised.,

\section{Tuberculosis of the appendix}

Abdominal TB complicates untreated pulmonary TB in $6 \%-38 \%$ of cases. ${ }^{5}$ Any organ of the gastrointestinal tract can have TB involvement, but the ileocecal region with its rich lymphoid tissue is the most common area involved. Three pathways have been described to cause occurrence of TB in the appendix: a direct lymphohematogenous route, an intestinal route and direct spread from peripheral organs. ${ }^{6}$ First described in 1837, appendicular TB is quite rare, with about 70 case reports published in English.

Foreign-born individuals from TB-endemic regions and Aboriginal Canadians have a higher risk of TB infection, as do close contacts of an individual with known TB disease. Tuberculosis should be considered in all patients with abdominal pain, fever and nonliver related ascites, if they fit the epidemiological profile noted above. ${ }^{5}$ Intraoperative clues may also be present. Tissues that are necrotic and have a "cottage-cheese" appearance similar to peritoneal carcinosis may indicate the presence of gastrointestinal and disseminated TB. ${ }^{5}$ Suspicion of TB appendicitis may also be raised by the patient's postoperative course. If, despite use of appropriate intravenous antibiot- 
ics and absence of an inflammatory focus, the patient continues to have prolonged peritoneal drain discharge and fever, TB involvement should be considered. ${ }^{8}$ The patient described in this case report had an uneventful postoperative course.

Granulomatous appendicitis is a rare entity, appearing in only $0.1 \%-2 \%$ of all appendectomy specimens. A four-year review of 3381 appendectomy specimens showed only 13 cases $(0.38 \%)$ of granulomatous appendicitis; of those, only one was believed to have been caused by TB. ${ }^{9}$ Other infectious causes for a granulomatous appendix include infection with Yersinia enterocolitica and parasitic infections (such as strongyloidiasis and enterobiasis), whereas noninfectious causes include Crohn disease, sarcoidosis, diverticulitis and foreign body. ${ }^{9}$

The treatment for appendicular TB is both surgical and medical. In conjunction with appendectomy, these patients must be given multiple antituberculous antibiotics as per standard guidelines. ${ }^{10}$ It is crucial that patients with appendicular or extrapulmonary TB be assessed for pulmonary involvement with chest radiography and sputum collection. This can help determine the risk of transmission to others, as well as guide therapeutic decisions and aid with diagnosis, as occurred in this case.

\section{References}

1. Tuberculosis in Canada 2012 - pre-release. Ottawa: Public Health Agency of Canada; 2012.

2. Global tuberculosis report 2013. Geneva: World Health Organization; 2013. Available: www.who.int/tb/publications /global_report/en/ (accessed 2014 Jan. 27).

3. Immigration medical examination instructions (IMEIs): tuberculosis. Ottawa: Citizenship and Immigration Canada; 2013. Available: www.cic.gc.ca/english/department/partner/pp/pdf /IMEI Tuberculosis.pdf (accessed 2014 Jan. 26).

4. Greenaway C, Sandoe A, Vissandjee B, et al. Tuberculosis: evidence review for newly arriving immigrants and refugees. CMAJ 2011;183:E939-51.

5. Cruz AT, Starke JR. Clinical manifestations of tuberculosis in children. Paediatr Respir Rev 2007;8:107-17.

6. Cruz AT, Ong L T, Starke JR. Emergency department presentation of children with tuberculosis. Acad Emerg Med 2011;18:726-32.

7. Ito N, Kawamoto S, Inada K, et al. Primary tuberculosis of the appendix in a young male patient: report of a case. Surg Today 2010;40:668-71.

8. Wyrobiec G, Sandelewski A, Glowacka M, et al. Acute isolated tuberculous appendicitis. J Clin Pharm Ther 2006;31:293-6.

9. AbdullGaffar B. Granulomatous disease and granulomas of the appendix. Int J Surg Pathol 2010;18:14-20.

10. Canadian tuberculosis standards, 7th edition 2013. Ottawa: Canadian Thoracic Society/Public Health Agency of Canada and Canadian Lung Association; 2013. Available: www.respiratory guidelines.ca/tb-standards-2013 (accessed 2014 Feb. 9).

Affiliations: Section of Infectious Diseases, Department of Pediatrics (Constantinescu, Vayalumkal), University of Calgary, Alberta Children's Hospital; Division of Respiratory Medicine, Department of Medicine, (Fisher) University of Calgary, Calgary, Alta.

Contributors: Cora Constantinescu drafted the article, which all of the authors revised and approved for publication.

Acknowledgement: The authors thank Alfredo Pinto, from the University of Calgary, for providing the pathology images and expertise for this manuscript.

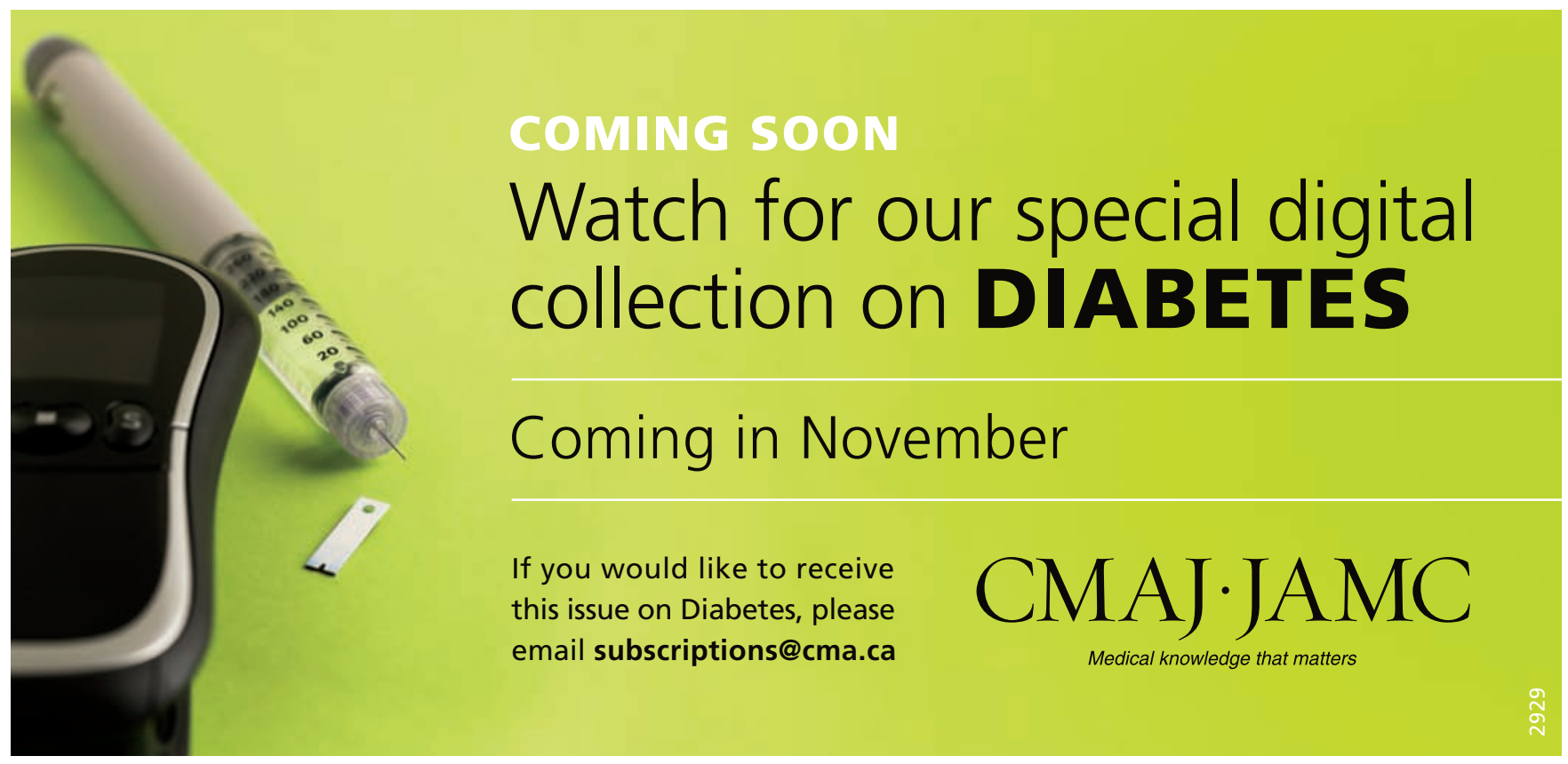

